\title{
Barmeyer, J.: Das kardiologische Gutachten; Anleitungen zur differenzierten Begutachtung bei Herz-Kreislauf-Erkrankungen
}

\author{
2. Auflage. 2009, 280 S., 56 Abb., 162 Tab., Thieme, \\ Stuttgart-New York 2010, gebunden, 129,95 $€$
}

\section{Stephan Becher}

Online publiziert: 6. Juli 2010

(c) Springer-Verlag 2010

Über 10 Jahren haben Gutachter auf die 2. Auflage gewartet. In dieser Zeit hat sich die Kardiologie wesentlich weiterentwickelt. Dieser Entwicklung haben die Autoren Rechnung getragen, indem sie die für die Begutachtung gängigen Untersuchungsverfahren, Spiroergometrie und Echokardiographie in das neue Werk eingepflegt haben. Wie schon in der 1. Auflage gefällt das Buch durch zahlreiche Tabellen und Abbildungen, die den gut lesbaren Text auch für den medizinischen Laien verständlich machen. Weiterführende Hinweise auf Untersuchungsmethoden wie das MRT, das nicht unbedingt zum gutachterlichen Repertoire des niedergelassenen Kollegen gehört, werden gegeben.

In 40 Kapiteln werden ohne Ausnahme alle kardiologisch relevanten Themen aufgegriffen (wobei das 42. Kapitel der 1. Auflage „Herzneurose“ anderweitig integriert wurde). Der Leser findet alle relevanten Inhalte in den krankheitsbezogenen Kapiteln. Ein mehr als ausführliches Stichwortverzeichnis ermöglicht ein schnelles Auffinden von Textstellen. Das moderne Layout gefällt durch seine ansprechende Darstellung. Erstmalig wurde auch ein Schwerpunkt auf den Bereich der privaten Versicherung gelegt und in die jeweiligen Kapitel eingearbeitet. Allerdings werden die Begriffe und Bedingungen hinsichtlich der Berufsunfähigkeitsversicherung nicht deutlich dargestellt bzw. zu den Begriffen MdE/GdB abgegrenzt. In diesem Bereich sollten Korrekturen vorgenommen werden, die vielleicht bald in der 3. Auflage in einem ganz hervorragenden Buch vorgenommen werden, so dass die Leserschaft nicht wieder 10 Jahre auf eine Neuauflage warten muss.

S. Becher $(\bowtie)$

AXA Lebensversicherung AG, Köln, Deutschland

e-mail: Stephan.Becher@AXA.DE 